\title{
Study of Increment Proportional Guidance Law for High Maneuvering Target
}

\author{
Yao Jun, Wei Zhou Fei and Wang Xin \\ Shenyang Ligong University, Shenyang 110159, China \\ yaojunjun@sina.com,sylaizh@163.com
}

\begin{abstract}
For the inadequacies of the classical proportional navigation law, the mathematical model of increment proportional navigation law is established in this paper. According to the mathematical model comes to attacking targets uniform linear motion trajectory simulation. The trajectory simulation of classical proportional navigation law and incremental proportional navigation law about attacking high maneuvering target are compared. Simulation results show that incremental proportional navigation law can reduce the meet time, and stabilize the missile desired normal overload beating phenomenon in the end of the curve, also it can hit the high maneuvering target in the design requirements, and its performance is superior to classical proportional navigation law.
\end{abstract}

Keywords: Increment proportional guidance law; High maneuvering target; Relative motion equations; Ballistics' characters

\section{Introduction}

Proportional guidance law is used extensively in missile guidance and control system, because guidance trajectory is straight fairly and the advantage of easy to implement on technology. However, the trajectory of classical proportional guidance law has obvious shortages. Despite the increase of proportionality coefficient can reduce meet time, but make the system a reduction in the ability to restrain noise and the enlargement of the required normal overload at the same time. Simultaneously, in the process of depicting the curve of meet time, we can find that under the request of high accuracy, the edge of curve, where the missile and the target meets, has the obvious phenomenon that beating acutely. It has bad influence for reliability of missile's component, accuracy of missile's guidance and control system, and attacking maneuvering target. While choosing additional increment appropriately and introducing it into classical proportional guidance law is able to solve these problems [1-3].

\section{Derivation of Relative Motion Equations}

In the three chapter, we introduced classical proportional guidance law, the guidance relative equation is $\dot{\sigma}=K \dot{q}, \mathrm{~K}$ is proportional coefficient. Increment proportional guidance law continues to use basic law of classical proportional guidance law, and use $\dot{q}$ to proceed guidance and control at the same, but introduces a constant additional increment $\omega$, which transform quondam guidance relation equation into $\dot{\sigma}=K \dot{q}+\omega$. The relative position of missile and target sill uses figure 1. Physical meaning of the graph symbols will not mention any longer here. According to the relationship between the relative motion of the missile and target and the mathematical model of classical proportional guidance law, we can 
get relative motion equations of missile and target in the condition of additional increment $\mathrm{t}[4-6]$.

$$
\left\{\begin{array}{l}
\frac{d r}{d t}=V_{T} \cos \eta_{T}-V \cos \eta \\
r \frac{d q}{d t}=V \sin \eta-V_{T} \sin \eta_{T} \\
q=\sigma+\eta \\
q=\sigma_{T}+\eta_{T} \\
\dot{\sigma}=K \dot{q}+\omega \\
n_{y}=\frac{V}{g} \dot{\sigma}=\frac{V}{g}(K \dot{q}+\omega)
\end{array}\right.
$$

Among them, ${ }^{n}{ }$ is required normal overload of missile.

\section{Analysis of Ballistics' Characters}

\subsection{Influence of $\omega$ to Meeting Time $t$}

In the modern air defense combat, it's no doubt that less time for encounter will make a defender in a favorable position. Meeting time ${ }^{t}$ describes the speed is fast or slow when missiles is close to the target, obviously can be represented with the change of $r$, the faster the $r$ reduces, the less the meeting time. Processing, 1, 3, 4 types in type (1), we can get

$$
\dot{r}=V_{T} \cos \left(q-\sigma_{T}\right)-V \cos (q-\sigma)
$$

By type (2), in the process of approaching, $\dot{r}<0, V>V_{T}$. We can get ,the increase of $\mathrm{K}$ makes ${ }^{t}$ smaller. For a better analysis of the influence of $\omega$ to $t_{\text {.Assuming that }} V, V_{T}, \sigma_{T}$ are constant, in the condition of $q$ is constant. Because of

$$
\left\{\begin{array}{l}
q>\sigma \\
\dot{\sigma}=K \dot{q}+\omega
\end{array}\right.
$$

So the introduction of $\omega$ must have influence to $\dot{r}$, then influences $t$. If we can choose $\omega$ appropriately to make $(q-\sigma)$ much smaller comparing to classical proportional guidance, thus $V \cos (q-\sigma)$ increases accordingly, $\dot{r}$ decreases accordingly, then $\dot{r}$ decreases. In this condition, if $\dot{q}<0$, we can deduce $\omega<0$.Conversely, if $\dot{q}>0$, thus, $\omega>0$ [7-8].

\subsection{Influence of $\omega$ to Required Normal Overload $n_{y}$}

Missile's normal overload $n_{y}$ is pointing to that missile requires normal overload when flies. According to the given ballistic, missile's required law to overload must meet the tactical technical requirement from tactical standpoint, missile to target maneuverability strong, must have a much larger ${ }^{n_{y}}$. From the point of view of designing and manufacturing, considering the structure of projectile, the normal work of the instrument and equipment, insisting on ${ }^{n_{y}}$ as small as possible.

$$
\text { Analyze } n_{y}=\frac{V}{g} \frac{d \sigma}{d t}=\frac{V}{g}\left(K \frac{d q}{d t}+\omega\right)
$$


Obviously, ${ }^{n_{y}}$ increases with the increase of $K$.For a better analysis of influence of $\omega_{\text {to }} n_{y}$, assuming $V, g, K$ aren't change, opposite with classical proportional guidance law. If $\omega \dot{q}$ have the same symbol, where easy to know that ${ }^{n_{y}}$ becomes larger correspondingly. If $\omega, \dot{q}$ have the same symbol, then ${ }^{n_{y}}$ becomes smaller. In

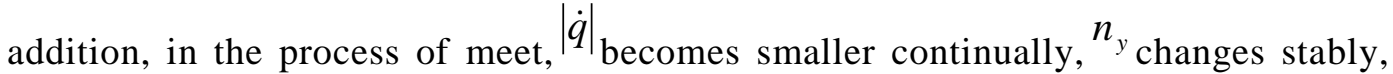
but in the near of missile's and target's encounter, if requirement of tracking,

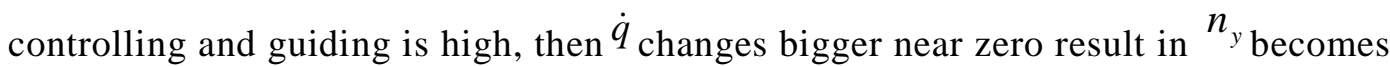
beating larger, normal overload acceleration is large, and then it is not conducive to the missile's accuracy of tracking and guiding. While choosing $\omega_{\text {reasonably will }}$ compensate ${ }^{n_{y}}$ near point of impact, and then reduce jitter which makes the edge of $n_{y}$, s curve change steadily[9-10].

\section{Analysis and Simulation of Increment Proportional Guidance Law}

\subsection{Simulation of Initial Condition}

Assuming that target makes uniform linear motion when flies in vertical plane, fight speed $V_{T}=350 \mathrm{~m} / \mathrm{s}$, target makes uniform linear motion when flies, missile's speed $V=1200 \mathrm{~m} / \mathrm{s}$, offensive plane is level. Missile's offensive requirement in the twinkling of starting guiding is that the distance of target and origin of the reference line $r_{\mathrm{o}}=4300 \mathrm{~m}$, goal line $q_{\mathrm{o}}=70^{\circ}$, leading angle $\sigma_{\mathrm{o}}=45^{\circ}$, then choose parameter appropriately, set up MATLAB simulation on above-mentioned mathematical model, angle change parameters model, then observe different simulation's results.

\subsection{Simulation and analysis of meeting time}

$\mathrm{We}$

set

additional

increment $\omega=[0.005,0,-0.01,-0.02,-0.03,-0.05,-0.1]$, proportionality coefficient $K=[2,3,4,5,6,7,10]$, and simulate in different case, then acquire the changing curve of meeting time ${ }^{t}$ in different conditions.

As is depicted in Figure 1, this is curve of meeting time with the change of proportionality coefficient when additional increment $\omega=0$.

We can find clearly from figure 1 that meeting time $t$ has the trend of decrease obviously with proportionality coefficient $\mathrm{K}$ increasing, but the trend becomes slower gradually with proportionality coefficient $\mathrm{K}$ become larger, when $K>7$, the decrease affection of $\mathrm{K}$ to $\mathrm{t}$ becomes dull gradually. At the same time, considering that the increase of proportionality coefficient will make required normal overload larger, therefore, we choose $\mathrm{K}$ among 3 6 universally.

Figure 2 researches influence of additional increment to meeting time $t$ when proportionality coefficient $\mathrm{K}$ is constant value, for figure (a) $\mathrm{K}=4$, for figure (b) $\mathrm{K}=7$. We can find that from the two figures, when additional increment $\omega$ is

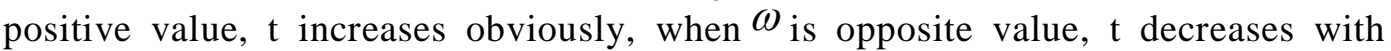

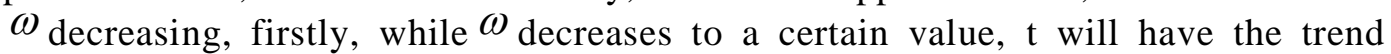
which increases gradually with decreasing again, it is mainly resulting from $V_{m} \cos \left(q-\eta_{m}\right)$ decreases when $\omega$ deceases a lot. It follows that it isn't so

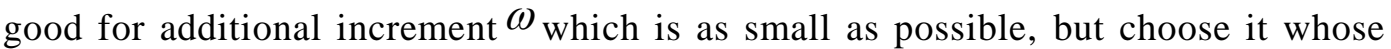


absolute value is small appropriately and generally, thus it will not increase required normal overload when decrease meeting time $t$.

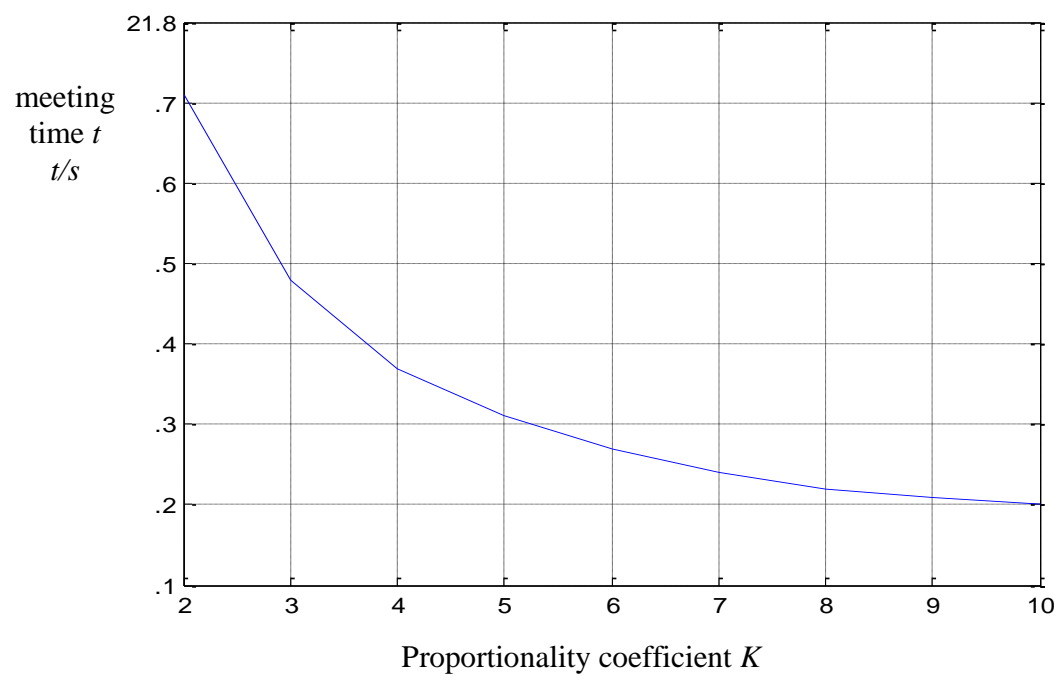

Figure 1. Curve of Meeting Time $t$ with Change of Proportionality Coefficient $K$ when Additional Increment $\omega=0$

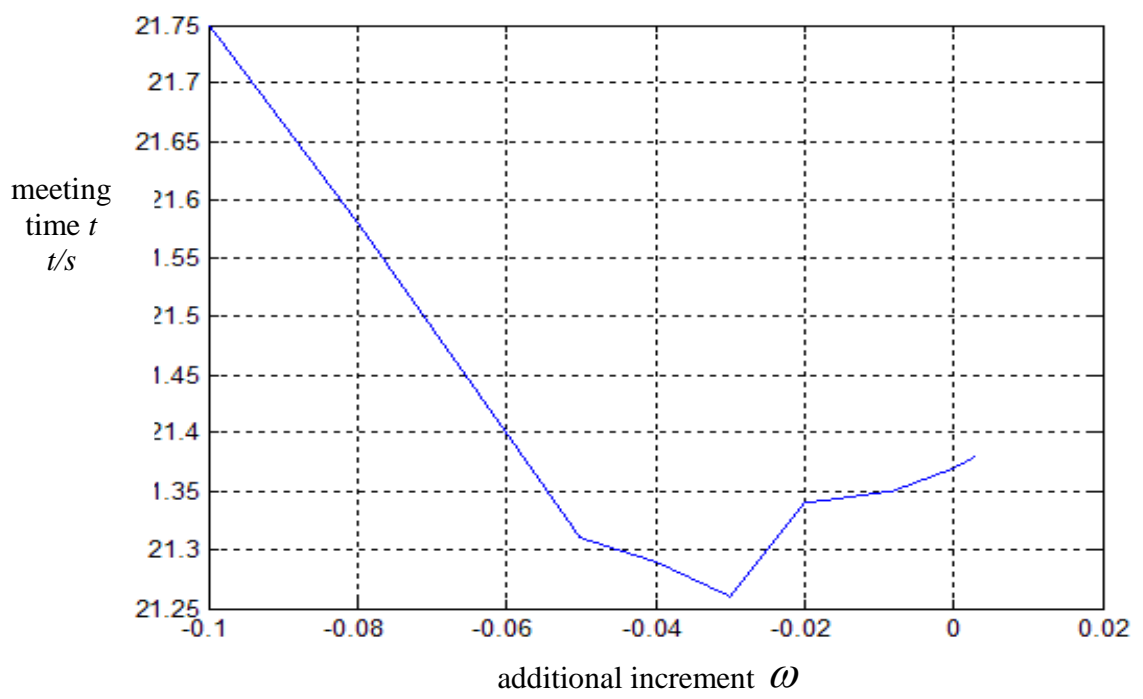

(a)

When $K=4$ 


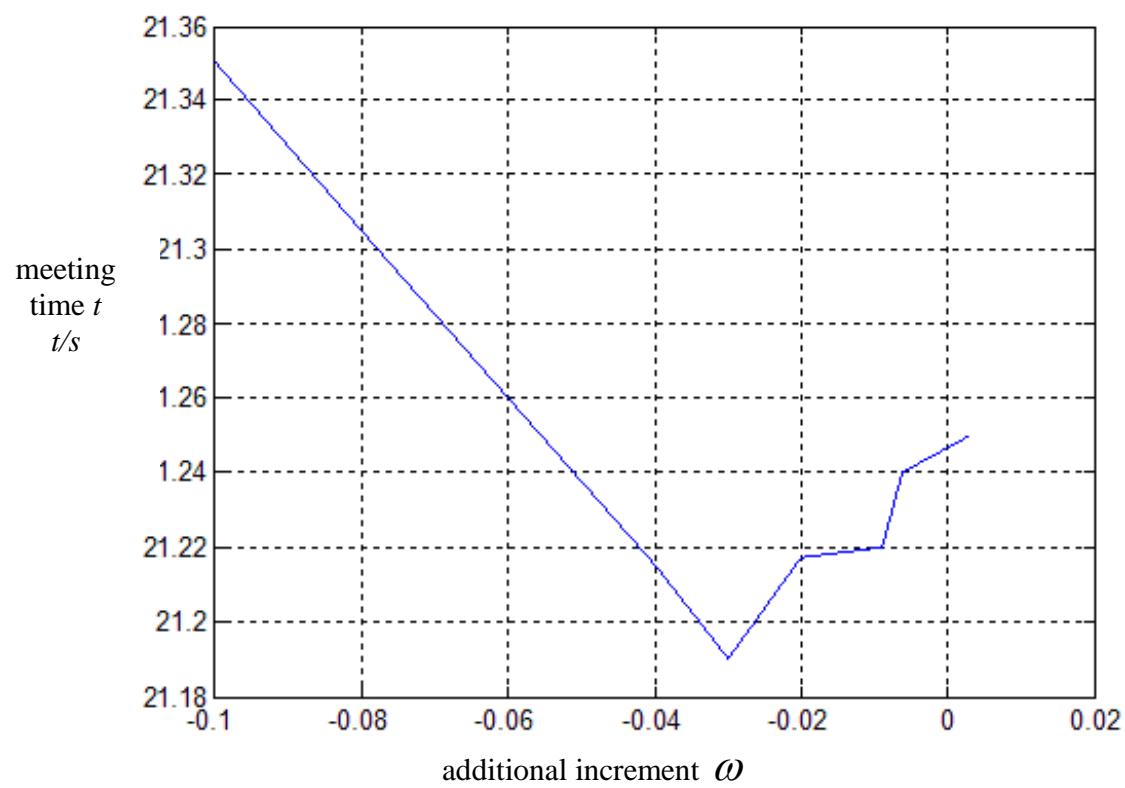

(b) When $K=7$

\section{Figure 2. Curve of Meeting Time with Change of Proportionality Coefficient when $K$ is Constant}

We can know that from Figure 1 and Figure 2 synthetically, choose additional increment $\omega$ appropriately can achieve the desired effect that the decrease of meeting time $t$ is as same as proportionality coefficient $\mathrm{K}$, thus the introduction of $\omega$ can decrease $\mathrm{K}$ accordingly in the case of $\mathrm{t}$ is smaller a litter, and solve the problem which the ability of suppressing noise of the large loop decreases and maximal required normal overload increases because $\mathrm{K}$ is larger a litter.

\subsection{Simulation and Analysis of Required Normal Overload}

In the case of introducing the simulation's principle of above, we choose proportionality coefficient $\mathrm{K}=[4,5,6]$, for the purpose of decreasing beating of the edge of required normal overload 's curve, and combine with Figure 2, let additional increment change in the interval $[0,-0.04]$ simulate, simulation graph is depicted as

Figure 3, a,b,c represent the curve of $n_{y}$ and els meeting time $\mathrm{t}$ when $\mathrm{K}=4,5,6$ respectively.

According to a,b,c in Figure 3, we can know that classical proportionality guidance $\operatorname{law}(\omega=0)$, the maximum of required normal overload $n_{y}$ increases obviously with proportionality coefficient K. For discretionary K, the edge of $n_{y}$,s curve beats bigger, the speed of $n_{y}$ changing faster a litter is not conducive to track targets stably for missile, more conducive to attack maneuvering targets. Choosing a appropriate value of $\omega$ can decrease beating of the edge of $n_{y}$, s curve obviously, and it will not lead to a larger required normal overload, then give the missile plenty of motor perform c, considering meeting time t. We can find that choose $\omega$ appropriately can not only decrease the phenomenon of beating, but also decrease meeting time $t$ relative to classical proportional guidance law, they always happen synchronously, it means that the affection of $\omega_{\text {to } t \text { and }} n_{y}$ happens synchronously. 
The above analyses the influence of proportional guidance coefficient and meeting time and required normal overload of missile through the above analysis, we can know that proportionality coefficient $\mathrm{K}$ and additional increment $\omega_{\text {have }}$ great influence meeting time $\mathrm{t} \mathrm{d}$ required normal overload ${ }^{n_{y}}$.In the case of proportionality coefficient is $\mathrm{K}$,choosing additional increment $\omega$ appropriately can decrease meeting time $t$, and it helps to stabilize the edge of 's curve and improve the shortages of classical proportional guidance law, which has important technical and tactical significance.

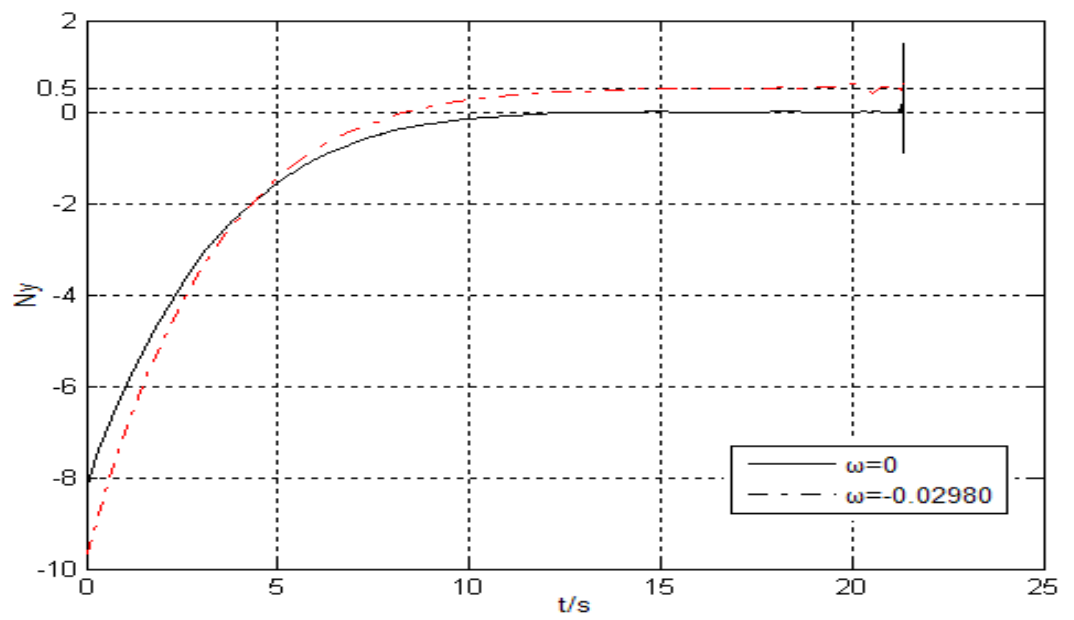

(a) when $K=4$

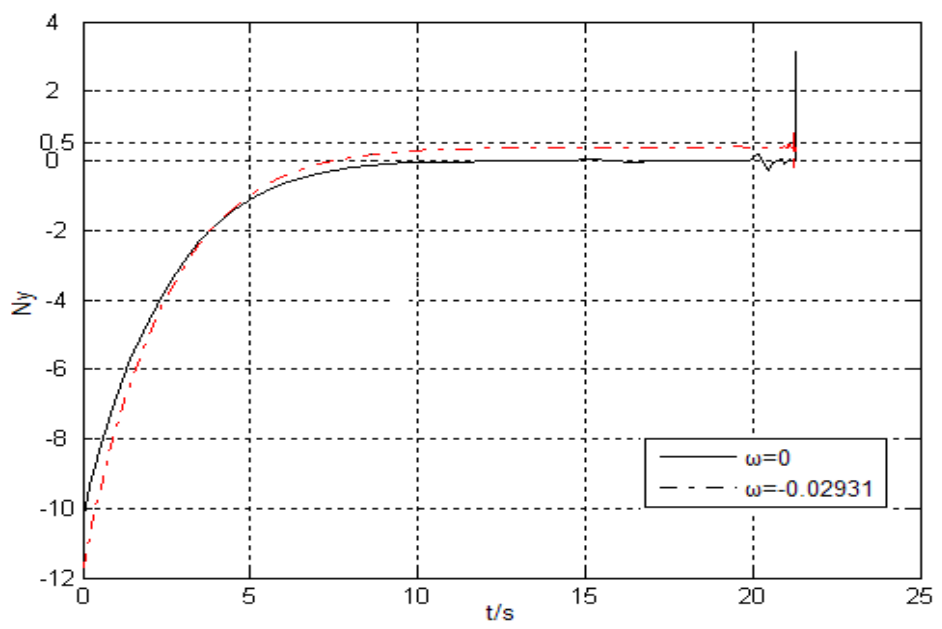

(b) when $K=5$ 


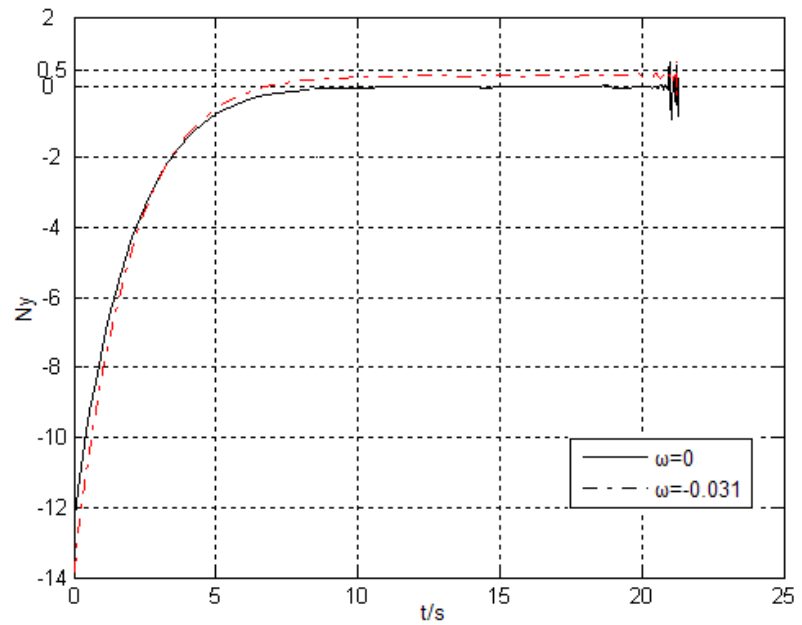

(c)when $K=6$

\section{Figure 3. Curve of $n_{y}$ and Meeting Time $t$ when $K$ is Changed with Different Value}

\section{Simulation and Design of Attacking Big Maneuvering Target}

\subsection{Maneuvering Targets}

In front of the targets is studied in uniform motion in a straight line of the classical proportional guidance law and incremental proportional guidance law of trajectory simulation, and understand classical proportional guidance law and incremental proportional guidance law preliminary. As a guidance law which is the most mature and applied widely, classical proportional guidance law is the better control and guidance law on the premise of the missile's and the target's speed is constant and the target does not have motor performance.

But in practice in order to avoid attacking, the distance features of maneuvering targets on the enemy's mobility. Maneuvering target will affect the impact on the performance of proportional guidance, which results in the increase of missing distance. Focus, the enemy of the maneuvering targets motion $y=f(t)$ is difficult to know in advance. We can only gain maneuvering targets motion through the methods of observation.

With the fast development of modern advanced technology, targets' maneuvering performance is more and more strong, its maximum could reach $60 \mathrm{~g}$.It appears weak on the high accuracy of control and guidance, the safeguard of precision applications relative to guided weapon of classical proportional guidance law.

In engineering applications, the maneuvering targets can be divided into three types roughly, the three typical mode of maneuvering targets are:

Maneuvering targets with constant acceleration, for example,

$a_{t}=n g$

The acceleration of maneuvering targets is sine change, for example,

$a_{t}=n g \sin (0.5 t)$

The acceleration of maneuvering targets is switching change, for example,

$a_{t}=n g \operatorname{sgn}(\sin ((t-5) \pi / 5))$

Among them, $\mathrm{n}=1 \sim 10, \mathrm{~g}=9.8 \mathrm{~m} / \mathrm{s} 2$

In the design of missile, required overload must satisfy the requirements of the missile, attacking maneuvering targets, the flying along a missile's guidance law requires that overload must be large. But considering the point of view of required 
overload's requirements of designing and manufacture, we feel like required overload could be as small as possible on the premise of meeting missile's tactical requirements, because the smaller the required overload, the smaller the supporting load when missile flights, it is very favorable to play on the normal work of the instrument, equipment and decrease the guidance error.

\subsection{Analysis of Comparison with Simulation}

Simulation conditions: assuming that target makes uniform linear motion when flies in vertical plane, fight speed $V_{T}=350 \mathrm{~m} / \mathrm{s}$, target makes uniform linear motion when flies, missile's speed $V=1200 \mathrm{~m} / \mathrm{s}$, offensive plane is level. Missile's offensive requirement in the twinkling of starting guiding is that the distance of target and origin of the reference line $r_{\mathrm{o}}=4300 \mathrm{~m}$, ,goal line $q_{\mathrm{o}}=70^{\circ}$, leading angle $\sigma_{0}=45^{\circ}$, set proportional coefficient $\mathrm{K}=4$, adopt mathematical model of the first two chapter, change model of parameters and set up simulating, observe simulation results.

Set the targets' motion equation as following:

Targets make parabola motion: (a is constant.)

$$
\left\{\begin{array}{l}
x_{T 1}=r_{0} \cos q_{0}+V_{T} \cdot t \\
z_{T 1}=r_{0} \sin q_{0}+\frac{1}{2} a t^{2}
\end{array}\right.
$$

$$
\left\{\begin{array}{l}
x_{T 2}=r_{0} \cos q_{0}+V_{T} \cos (t) \\
z_{T 2}=r_{0} \sin q_{0}+V_{T} \sin (t)
\end{array}\right.
$$

(a) and (b) in Figure 4 are ballistic trajectory comparison chart of increment proportional guidance law and classical proportional guidance law when additional increment $\omega=0.2$ and $\omega=0.4$.According to a and $\mathrm{b}$ of figure 4 , we can know that when maneuvering targets make parabola motion in the case of parameters is constant, ballistic trajectory of classical proportional guidance law is fixed, ballistic trajectory but increment proportional guidance law changes ballistic trajectory under the accommodation of additional increment, adopt additional increment appropriately could make ballistic trajectory missile more straight ,decrease required normal overload of missile sequentially.

(a) and (b) in figure 5 are ballistic trajectory comparison chart of increment proportional guidance law and classical proportional guidance law when additional increment $\omega=-0.12$ and $\omega=0.2$.According to a and $\mathrm{b}$ of figure 5 , we can know that when maneuvering targets make parabola motion in the case of parameters is constant, ballistic trajectory of classical proportional guidance law is fixed, ballistic trajectory but increment proportional guidance law changes ballistic trajectory under the accommodation of additional increment, adopt additional increment appropriately could make ballistic trajectory missile more straight, in addition, it is able to decrease changing frequency of ballistic trajectory's curvature. It means that additional increment is able to decrease required normal overload of missile, at the same time, it can decrease changing frequency. 


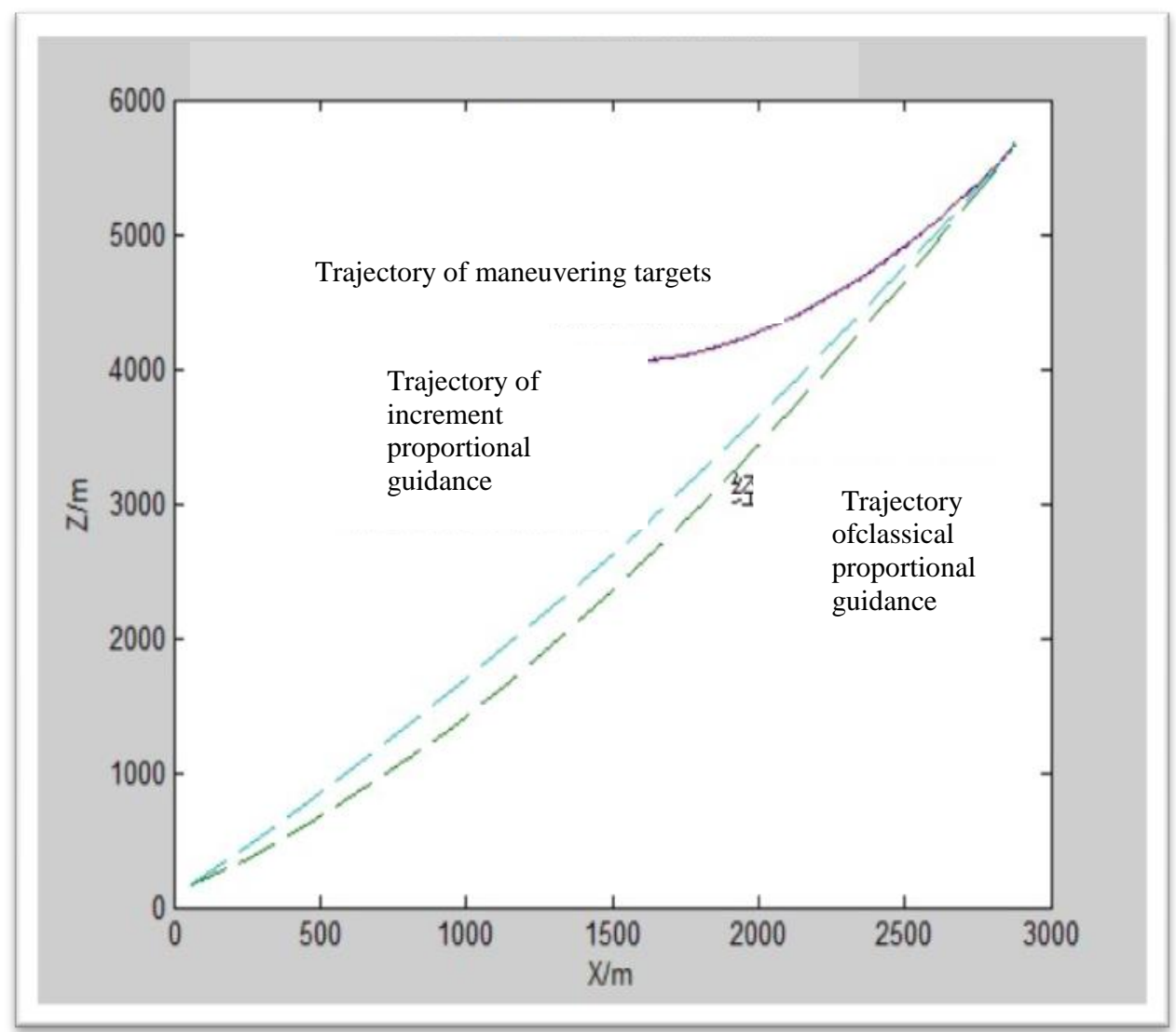

(a) when $\omega=0.2, \mathrm{~K}=4$

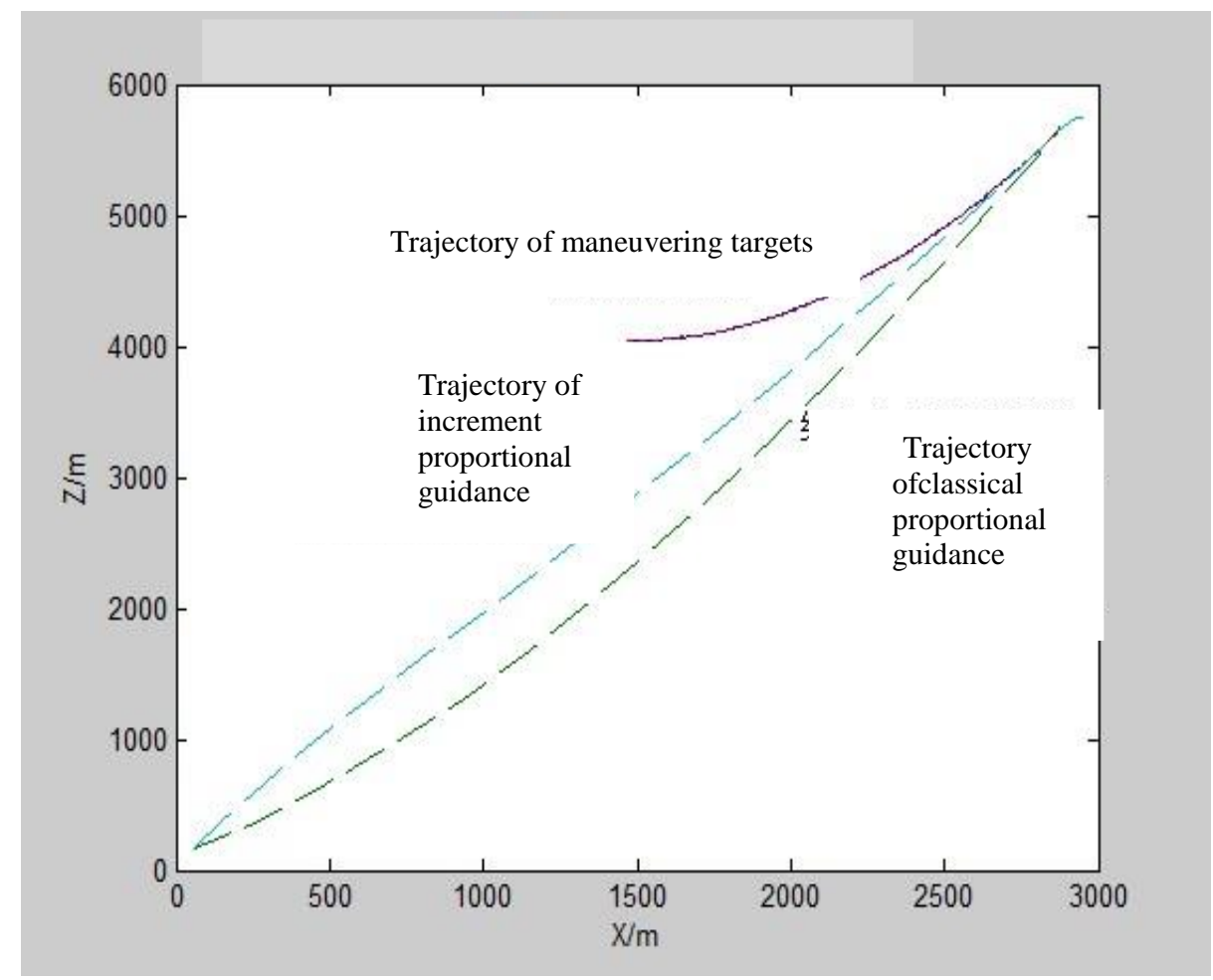

(b) when $\omega=0.4, \mathrm{~K}=4$

Figure 4. Simulation and Comparison when Target Makes Parapola Motion 


\section{Conclusions}

In the paper, we simulate, compare and analyze the trajectory of classical proportional guidance law and increment proportional guidance law when missiles attack big maneuvering targets in the case of basic parameters are not change through the introduction and analysis of maneuvering targets. The simulation results

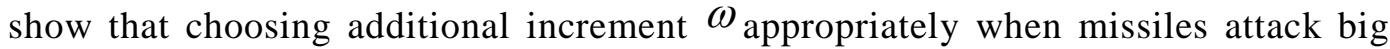
maneuvering targets can not only decrease required normal overload of missile, but also decrease changing frequency of normal overload, which makes ballistic trajectory missile more straight it helps to stability of trajectory and control of missile's required normal overload, and protect the normal work of the projectile bodies' structure and precision instrument effectively, reduce the guidance error, and is more easy to hit big maneuvering targets. Therefore increment proportional guidance law has more advantages and reference value relative to classical proportional guidance law aiming at attacking big maneuvering targets, and it can meet the design requirements preferably.

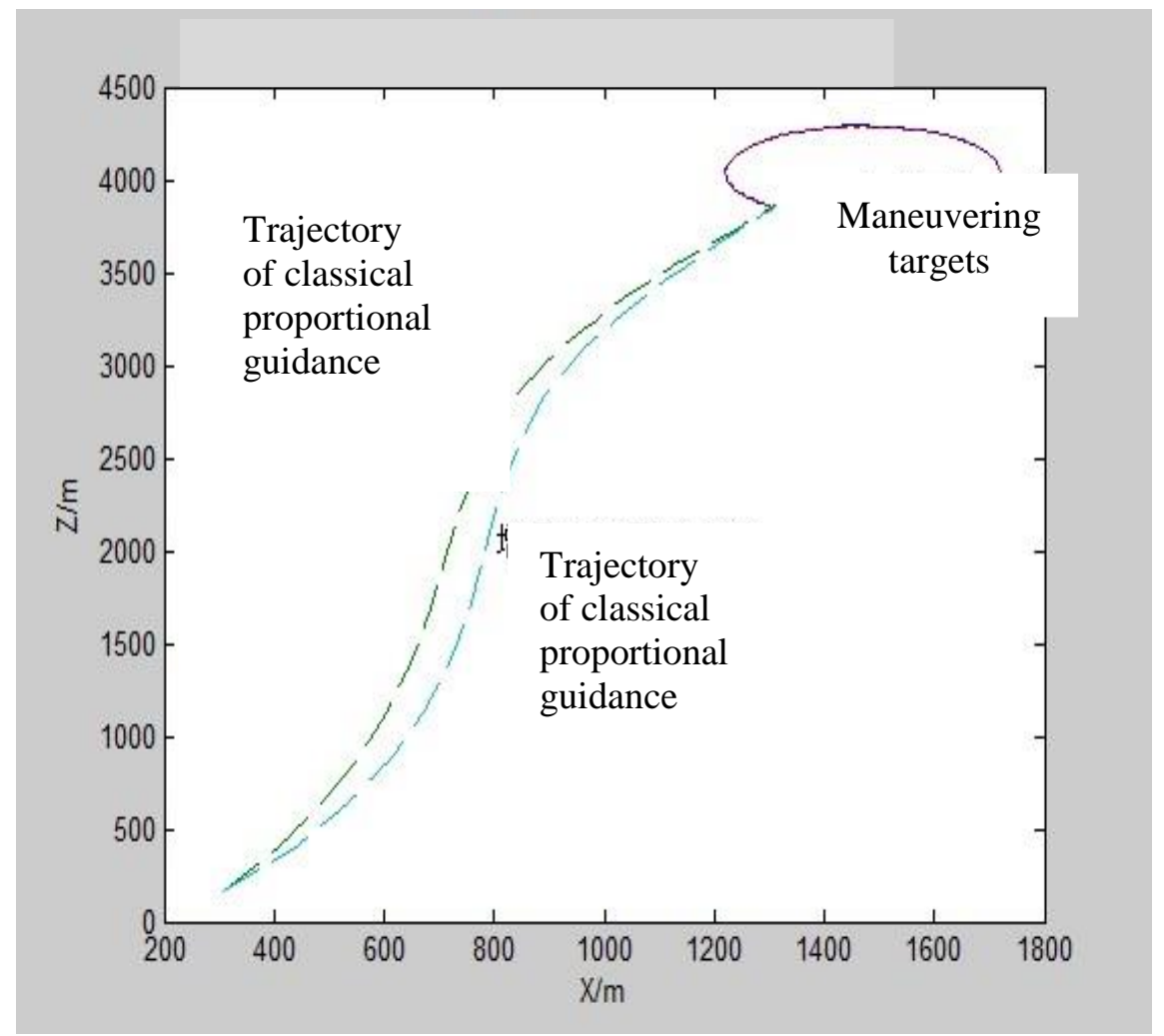

(a) when $\omega=-0.12, \mathrm{~K}=4$ 


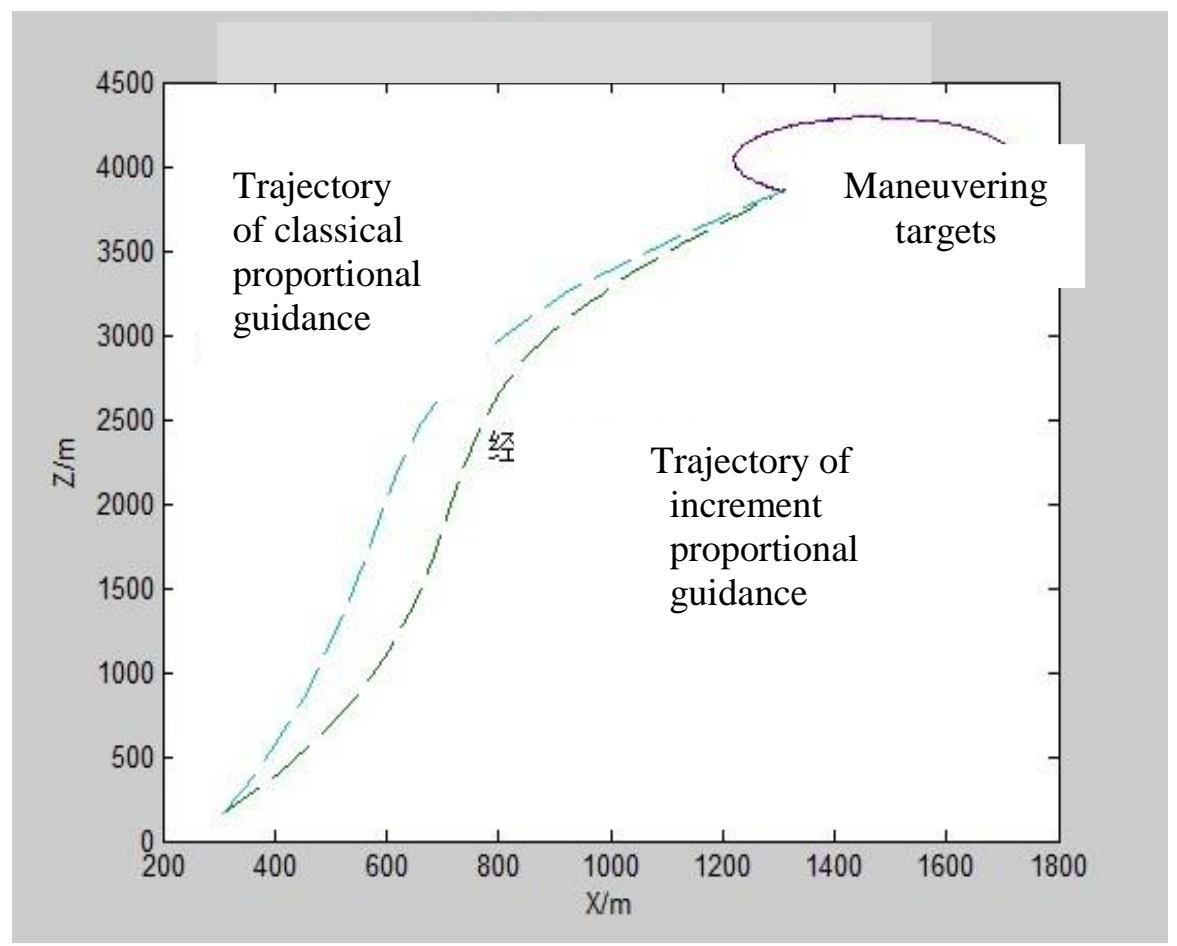

(b) when $\omega=0.2, \mathrm{~K}=4$

Figure 5. Simulation and Comparison as the Target Makes Helical Motion

\section{References}

[1] Y. Zhang and P. B. Ma, "Three-dimensional guidance law with impact angle and impact time constraints", Acta Aeronaut. Astronaut. Sin, vol. 29, no. 4, (2008), pp. 1020-1026.

[2] J. M. Song and T. Q. Zhang, "The passive homing missile's variable structure guidance law with terminal impact angular costraints", J. Ballist., vol. 13, no. 1, (2001), pp. 16-21.

[3] S. B. Cao, C. S. Jiang, S. Y. Guang and M. Chen, "Research on the vertical intercept terminal guidance system of TV-command-guidance air-to-ground missile", J. Astronaut., vol. 25, no. 4, (2004), pp. 393397.

[4] Y. X. Yin, M. Yang and P. Wu, "Three dimensional guidance laws with attack angle constraint for maneuverable target", Solid Rocket, Technol., vol. 33, no. 3, (2010), pp. 237-241.

[5] Z. Zhang, Yo. Tong and W. Zhang, "Research on the Proportional Guidance Trajectory Simulation [J]", Tactical Missile Technology, vol. 2, (2005), pp. 56-59.

[6] J. Zhou, Y. Tong and L. Zhang, "Simulation and Analysis of Three-dimensional Trajectory on Typical Guide Method [J]", Ship Electronic Engineering, vol. 2, (2008), pp. 110-112.

[7] S. Gao, "Proportional Navigation ideal trajectory simulation [J]", Computer Engineering and Design, vol. 24, no. 8, (2003), pp. 66-67.

[8] Y.-f. Wang, Y.-w. Fang and X.-b. Zhou, "Proportional Navigation Research and Development [J]", Fire Control and Command Control, vol. 32, no. 10, (2007), pp. 8-11.

[9] D. Zhou and S. Sun, "Guidance laws with finite time convergence", J. Guid. Control Dyn., vol. 32, no. 6, (2009), pp. 1838-1846.

[10] S. Sun, D. Zhou and W. T. Hou, "A guidance law with finite time convergence accounting for autopilot lag. Aerosp. Sci.Technol.,” (2012, in press). doi:10.1016/j.ast.2011.12.016 
International Journal of Signal Processing, Image Processing and Pattern Recognition Vol.8, No.6 (2015) 